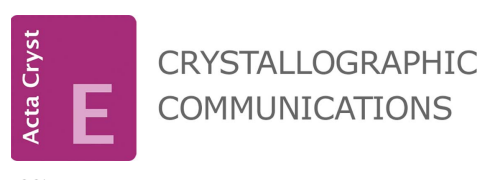

ISSN 2056-9890

Received 2 March 2017

Accepted 9 March 2017

Edited by P. C. Healy, Griffith University, Australia

Keywords: crystal structure; tellurium; Hirshfeld surface analysis; heavy-atom chirality.

CCDC reference: 1537011

Supporting information: this article has supporting information at journals.iucr.org/e

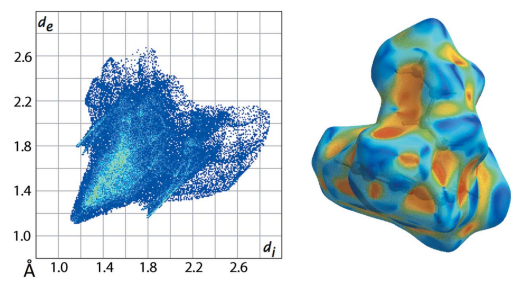

OPEN $\odot$ ACCESS

\section{1-Butyl-1-chloro-3-methyl-3H-2,1 $\lambda^{4}$-benzoxa- tellurole: crystal structure and Hirshfeld analysis}

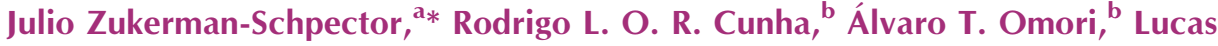 \\ Sousa Madureira ${ }^{\mathrm{a}}$ and Edward R. T. Tiekink ${ }^{\mathrm{c}}$
}

\begin{abstract}
${ }^{a}$ Departmento de Química, Universidade Federal de São Carlos, 13565-905 São Carlos, SP, Brazil, bentro de Ciências Naturais e Humanas, Universidade Federal do ABC, Av. Dos Estados 5001, Bairro Bangu, CEP 09210-580 Santo André, SP, Brazil, and ${ }^{\mathrm{c} C e n t r e ~ f o r ~ C r y s t a l l i n e ~ M a t e r i a l s, ~ S c h o o l ~ o f ~ S c i e n c e ~ a n d ~ T e c h n o l o g y, ~ S u n w a y ~ U n i v e r s i t y, ~} 47500$ Bandar Sunway, Selangor Darul Ehsan, Malaysia. *Correspondence e-mail: julio@power.ufscar.br
\end{abstract}

Two independent molecules comprise the asymmetric unit in the title benzoxatellurole compound, $\mathrm{C}_{12} \mathrm{H}_{17} \mathrm{ClOTe}$. The molecules, with the same chirality at the methine $\mathrm{C}$ atom, are connected into a loosely associated dimer by $\mathrm{Te} \cdots \mathrm{O}$ interactions, leading to a $\{\cdots \mathrm{Te}-\mathrm{O}\}_{2}$ core. The resultant $\mathrm{C}_{2} \mathrm{ClO}_{2}$ donor set approximates a square pyramid with the lone pair of electrons projected to occupy a position trans to the $n$-butyl substituent. Interestingly, the $\mathrm{Te}^{\mathrm{IV}}$ atoms exhibit opposite chirality. The major difference between the independent molecules relates to the conformation of the five-membered chelate rings, which is an envelope with the $\mathrm{O}$ atom being the flap, in one molecule and is twisted about the $\mathrm{O}-\mathrm{C}($ methine) bond in the other. No directional intermolecular interactions are noted in the molecular packing beyond the aforementioned $\mathrm{Te}$... O secondary bonding. The analysis of the Hirshfeld surface reveals the dominance of $\mathrm{H} \cdots \mathrm{H}$ contacts, i.e. contributing about $70 \%$ to the overall surface, and clearly differentiates the immediate crystalline environments of the two independent molecules in terms of both $\mathrm{H} \cdots \mathrm{H}$ and $\mathrm{H} \cdots \mathrm{Cl} / \mathrm{Cl} \cdots \mathrm{H}$ contacts.

\section{Chemical context}

Tellurium is not the first element that comes to mind when considering the modern pharmacopoeia (Tiekink, 2012). However, investigations into pharmaceutical applications of compounds of this generally regarded as relatively non-toxic element (Nogueira et al., 2004) date back to the times of Sir Alexander Fleming who tested the efficacy of potassium tellurite, $\mathrm{K}_{2}\left[\mathrm{TeO}_{3}\right]$, against microbes, such as penicillin-insensitive bacteria (Fleming, 1932). It is in fact another salt, ammonium trichloro(dioxyethylene-O, $\left.\mathrm{O}^{\prime}\right)$ tellurate, $\left[\mathrm{NH}_{4}\right]\left[\left(\mathrm{OCH}_{2} \mathrm{CH}_{2} \mathrm{O}\right)\right.$ $\mathrm{TeCl}_{3}$ ] (Albeck et al., 1998), also known as AS-101, that has attracted the most attention as a potential tellurium-based pharmaceutical, being in clinical trials for the treatment of psoriasis (Halpert \& Sredni, 2014). Other potential applications of AS-101 include its use as an anti-inflammatory agent (Brodsky, et al., 2010), as a topical treatment for human papilloma virus (Friedman et al., 2009) and its ability to inhibit angiogenesis (Sredni, 2012). The anti-cancer potential of tellurium compounds has also attracted attention (Seng \& Tiekink, 2012; Silberman et al., 2016). The cation in AS-101 has long been known to be a specific inhibitor of both papain and cathepsin $\mathrm{B}$, i.e. cysteine proteases, by forming a covalent $\mathrm{Te}-\mathrm{S}$ (cysteine) bond (Albeck et al., 1998). Organotellurium compounds also inhibit cathepsin B (Cunha et al., 2005) and docking studies confirm this hypothesis (Caracelli et al., 2012, 
2016). It was in this context that the title compound, (I), was prepared. Herein, the crystal and molecular structures of (I) are described as well as an analysis of its Hirshfeld surface. Finally, a preliminary inhibition assay on (I) against cathepsin $\mathrm{B}$ has been performed.

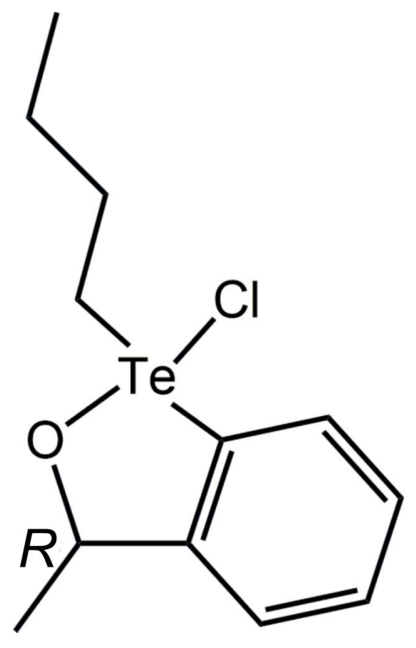

\section{Structural commentary}

The asymmetric unit of (I) comprises two independent molecules, which are connected into a loosely associated dimer via secondary Te...O interactions, as shown in Fig. 1. The immediate geometry for the $\mathrm{Te}^{\mathrm{IV}}$ atom in the Te1-containing molecule is defined by chlorido, oxygen and carbon (within the oxatellurole ring) and $n$-butyl alpha-carbon atoms. While the bridging-O2 atom forms a significantly longer Te...O2 bond than the $\mathrm{Te}-\mathrm{O} 1$ bond, Table 1 , it must be included in the coordination geometry, which is then best described as being distorted square pyramidal. This arrangement accommodates a stereochemically active lone-pair of electrons in the position trans to the $n$-butyl group. The coordination geometry for the Te2-containing molecule is essentially the same.

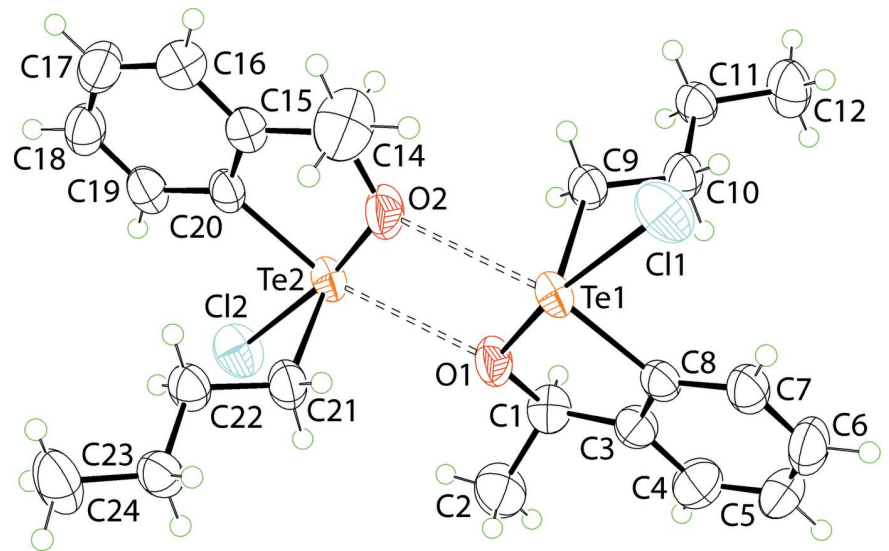

Figure 1

The molecular structures of the two independent molecules comprising the asymmetric unit of (I), showing the atom-labelling scheme and displacement ellipsoids at the $50 \%$ probability level. The molecules associate via secondary Te... O bonding shown as dashed bonds.
Table 1

Selected geometric parameters $\left(\AA,^{\circ}\right)$.

\begin{tabular}{lclc}
\hline $\mathrm{Te} 1-\mathrm{Cl} 1$ & $2.6137(17)$ & $\mathrm{Te} 2-\mathrm{Cl} 2$ & $2.5944(17)$ \\
$\mathrm{Te} 1-\mathrm{O} 1$ & $2.021(4)$ & $\mathrm{Te} 2-\mathrm{O} 2$ & $2.010(5)$ \\
$\mathrm{Te} 1-\mathrm{C} 8$ & $2.107(6)$ & $\mathrm{Te} 2-\mathrm{C} 20$ & $2.108(6)$ \\
$\mathrm{Te} 1-\mathrm{C} 9$ & $2.138(5)$ & $\mathrm{Te} 2-\mathrm{C} 21$ & $2.136(6)$ \\
$\mathrm{Te} 1-\mathrm{O} 2$ & $2.945(4)$ & $\mathrm{Te} 2-\mathrm{O} 1$ & $2.977(4)$ \\
& & & \\
$\mathrm{Cl} 1-\mathrm{Te} 1-\mathrm{O} 1$ & $171.04(13)$ & $\mathrm{Cl} 2-\mathrm{Te} 2-\mathrm{O} 2$ & $170.22(14)$ \\
$\mathrm{O} 1-\mathrm{Te} 1-\mathrm{C} 8$ & $80.4(2)$ & $\mathrm{O} 2-\mathrm{Te} 2-\mathrm{C} 20$ & $80.5(2)$ \\
$\mathrm{C} 8-\mathrm{Te} 1-\mathrm{O} 2$ & $145.0(2)$ & $\mathrm{C} 20-\mathrm{Te} 2-\mathrm{O} 1$ & $145.38(19)$ \\
\hline
\end{tabular}

The bond lengths about the $\mathrm{Te}^{\mathrm{IV}}$ atoms in the independent molecules are similar, Table 1 . However, the Te1-Cl1 bond length is longer by approximately $0.02 \AA$ than the chemically equivalent $\mathrm{Te}-\mathrm{Cl} 2$ bond. The three remaining 'short' bond lengths are equal within experimental error. The disparity in the $\mathrm{Te}-\mathrm{Cl}$ bond lengths is probably compensated by the Te...O secondary bond, which is shorter, by approximately $0.03 \AA$, in the Te1-molecule. The key pairs of bond angles for the molecules are essentially the same with the major difference, i.e. $0.8^{\circ}$, seen in the $\mathrm{Cl}-\mathrm{Te}-\mathrm{O}_{\text {long }}$ angle. A distinguishing feature of the independent molecules is noted in the conformation of the five-membered, chelate rings. Thus, in the Te1-molecule, the chelate ring has the form of an envelope with the flap atom being the $\mathrm{O} 1$ atom [the $\mathrm{O} 1$ atom lies 0.254 (8) $\AA$ out of the plane through the remaining atoms; r.m.s. deviation $=0.0107 \AA]$. For the Te2-molecule, the chelate ring is twisted about the $\mathrm{O} 2-\mathrm{C} 13$ bond, as seen in the $\mathrm{Te} 2-$ $\mathrm{O} 2-\mathrm{C} 13-\mathrm{C} 15$ torsion angle of 12.1 (7) ${ }^{\circ}$.

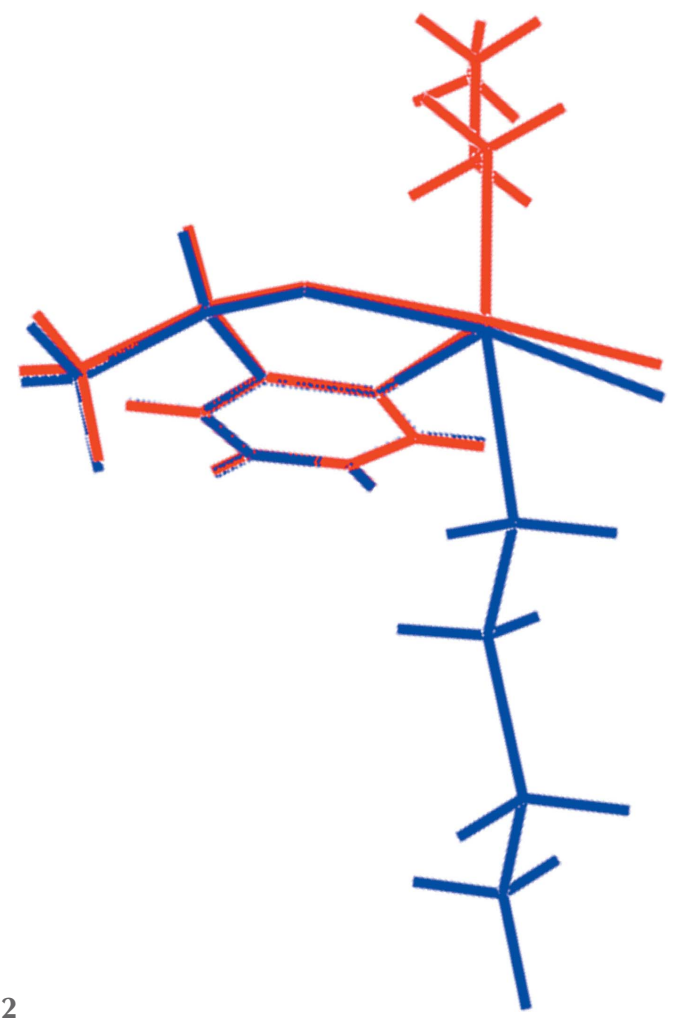

Figure 2

An overlay diagram of the Te1- and Te2-containing molecules, shown as red and blue images, respectively. The molecules have been overlapped so that the phenyl rings are coincident. 
Table 2

Percentage contributions of the different intermolecular contacts to the Hirshfeld surface in (I), Te1-molecule in (I) and Te2-molecule in (I).

\begin{tabular}{llll}
\hline Contact & overall (I) & Te1-molecule in (I) & Te-2 molecule in (I) \\
\hline $\mathrm{H} \cdots \mathrm{H}$ & 70.3 & 65.1 & 66.2 \\
$\mathrm{H} \cdots \mathrm{C} \cdots \mathrm{l} / \mathrm{Cl} \cdots \mathrm{H}$ & 16.6 & 15.7 & 15.4 \\
$\mathrm{H} \cdots \pi / \pi \cdots \mathrm{H}$ & 5.5 & 4.1 & 4.2 \\
$\mathrm{Te} \cdots \pi / \pi \cdots \mathrm{Te}$ & 4.0 & 3.7 & 3.6 \\
$\mathrm{H} \cdots \mathrm{Te} / \mathrm{Te} \cdot \mathrm{H}$ & 0.4 & 3.3 & 2.6 \\
$\mathrm{H} \cdots \mathrm{O} / \mathrm{O} \cdots \mathrm{H}$ & 0.0 & 2.9 & 2.9 \\
$\mathrm{O} \cdots \mathrm{Te} / \mathrm{Te} \cdots \mathrm{O}$ & 0.0 & 1.7 & 1.6 \\
$\pi-\pi / \pi-\pi$ & 1.7 & 1.5 & 1.5 \\
Others & 1.5 & 2.0 & 2.0 \\
\hline
\end{tabular}

The central $\{\cdots \mathrm{Te}-\mathrm{O}\}_{2}$ core of the dimeric aggregate, Fig. 1 , is almost planar (r.m.s. deviation $=0.0106 \AA$ ) and has the form of a parallelogram with distinctive edge lengths of approximately 2.0 and $3.0 \AA$ A, reflecting the disparity of the Te... interactions. To a first approximation, the fused phenyl ring in each molecule, (C3-C8) and (C13-C20), is co-planar with the core, forming dihedral angles of $14.2(2)$ and $13.6(3)^{\circ}$, respectively; the dihedral angle between the phenyl rings is $8.3(3)^{\circ}$. As the $n$-butyl groups lie to either side of the dimeric aggregate, there is a suggestion that the independent molecules are related across a pseudo centre of inversion. However, the configuration of the chiral- $\mathrm{C} 2$ and $\mathrm{C} 13$ atoms in the Te1- and Te-molecules, respectively, is $R$. This is highlighted in the overlay diagram shown in Fig. 2. Also highlighted is that the tellurium atoms have opposite chirality. When projected down the $\mathrm{Te}-\mathrm{C}(n$-butyl $)$ bond, the chirality about the Te1 atom is $S$ and that about Te2, $R$.

\section{Supramolecular features}

Beyond the secondary Te... O secondary contacts, leading to dimeric aggregates, Fig. 1, no directional interactions, according to the criteria in PLATON (Spek, 2009), are apparent in the crystal of (I). A view of the unit-cell contents is shown in Fig. 3.

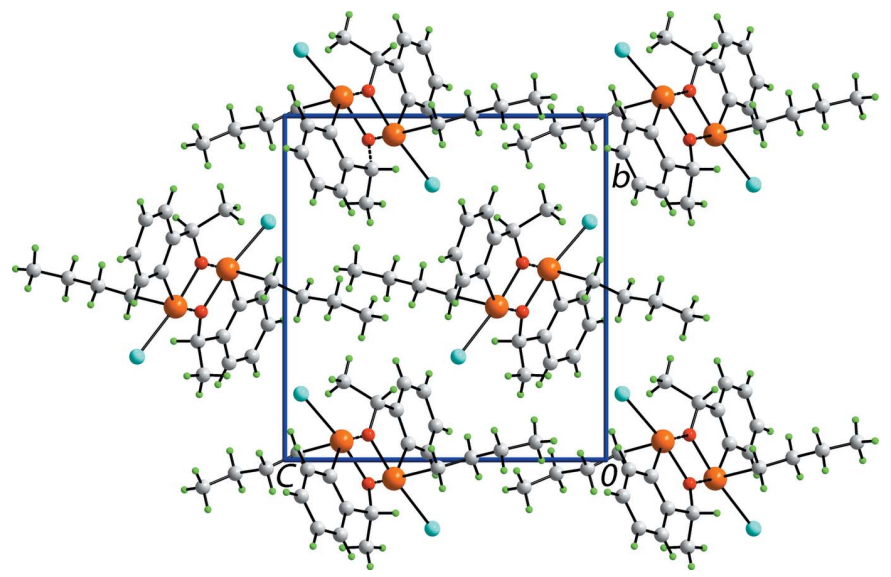

Figure 3

A view in projection down the $a$ axis of the molecular packing in (I).

\section{Hirshfeld surface analysis}

An analysis of the Hirshfeld surface for (I) was conducted using protocols established earlier (Jotani et al., 2016). The overall two-dimensional fingerprint plot for the asymmetric unit is shown in Fig. $4 a$ and those for the individual Te1- and Te2-containing molecules are shown in Fig. $4 b$ and $c$. The shape-index surface properties are also illustrated in Fig. 4. These confirm the absence of significant directional interactions in the crystal.

Referring to Fig. 5 and Table 2, the Hirshfeld surface is dominated by $\mathrm{H} \cdots \mathrm{H}$ interactions, contributing around $70 \%$ to the overall surface of the asymmetric unit and about $65 \%$ for each independent molecule. While not within the sum of the respective van de Waals radii, the $\mathrm{C}-\mathrm{H} \cdots \mathrm{Cl}$ contacts make the next greatest contribution to the overall surface, i.e. $c a$ $15 \%$. Others interactions each contribute less than $5 \%$ to the

(a)
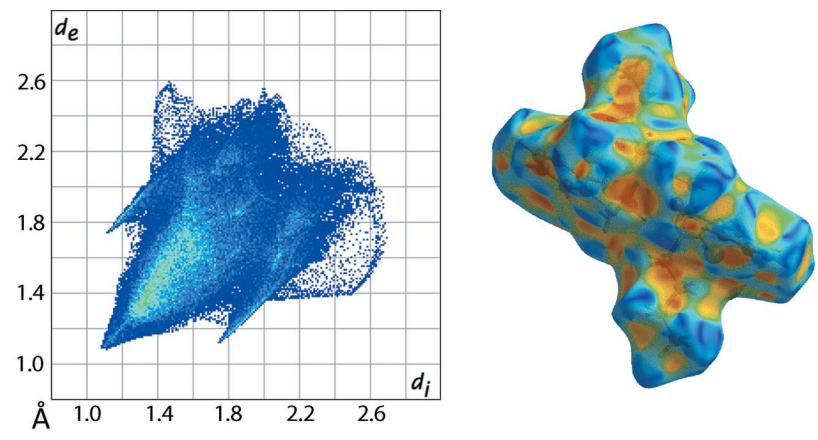

(b)
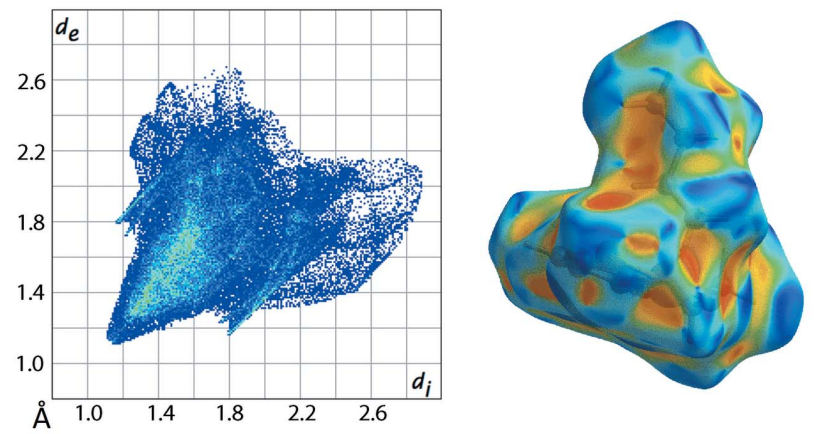

(c)
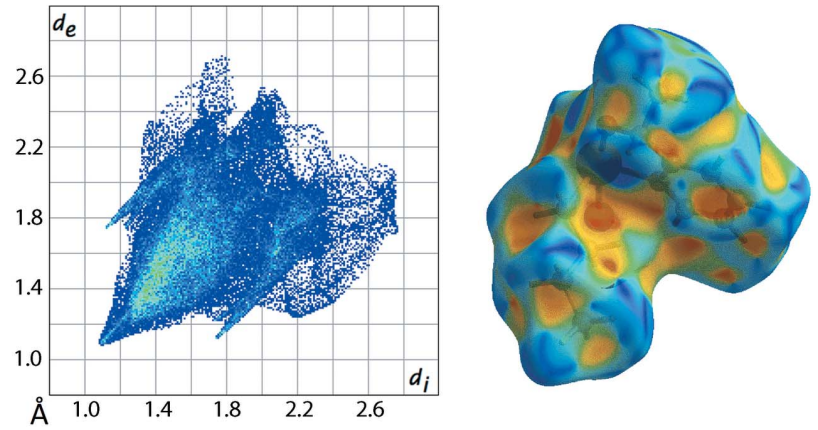

Figure 4

Two-dimensional fingerprint plots and shape index surface properties of the Hirshfeld surface analysis for $(a)(\mathrm{I}),(b)$ the Te1-molecule in (I) and (c) the Te2-molecule in (I). 


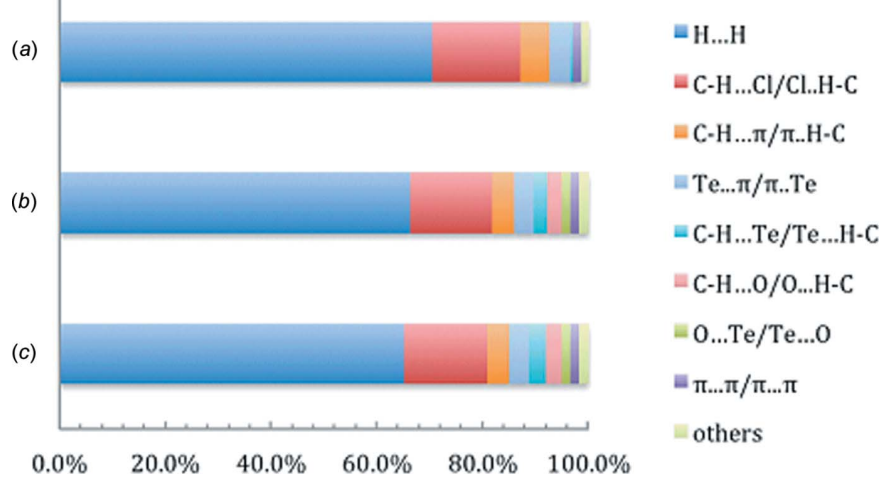

Figure 5

Charts of the relative percentage contributions of the intermolecular contacts to the Hirshfeld surface area for $(a)(\mathrm{I}),(b)$ the Te1-molecule in (I) and (c) the Te2-molecule in (I).

Hirshfeld surface. It should be noted that the $\mathrm{C}-\mathrm{H} \cdots \mathrm{O}$ contacts, $\mathrm{Te} \cdots \mathrm{O}$ secondary interactions and most of the $\mathrm{C}-$ $\mathrm{H}$. . Te contacts are formed between the two independent molecules, thus they are overlapped and do not contribute to surface area of the asymmetric unit.

The main differences between the surface areas of the independent molecules are in the interactions of the type $\mathrm{H} \cdots \mathrm{H}$ and $\mathrm{C}-\mathrm{H} \cdots \mathrm{Cl}$. Referring to Fig. 6, the red circles on the fingerprint plots delineated into $\mathrm{H} \cdots \mathrm{H}$, Fig. $6 a$ and $\mathrm{H} \cdots \mathrm{Cl} / \mathrm{Cl} \cdots \mathrm{H}$ contacts, Fig. $6 b$, highlight the distinctive features of the interactions for the two molecules. For example, short $\mathrm{H} \cdots \mathrm{H}$ interactions for the Te2-molecule, (a)

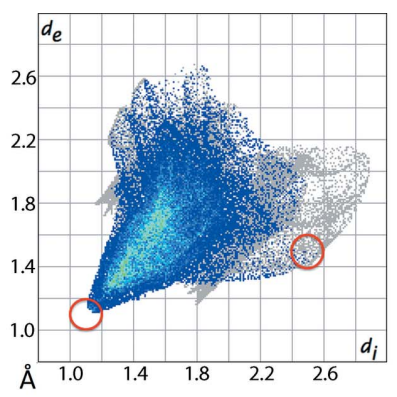

(b)

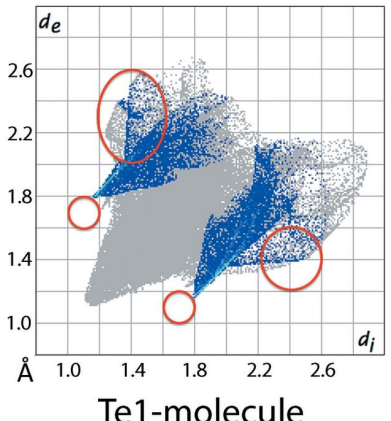

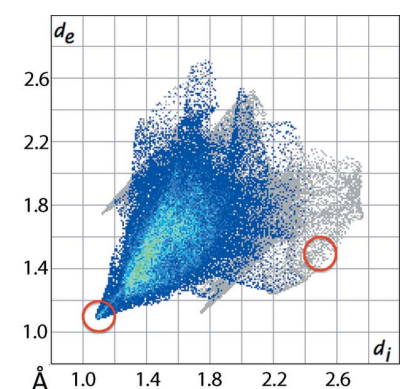

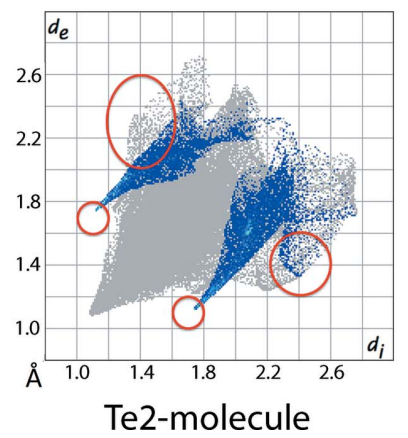

Figure 6

Two-dimensional fingerprint plots delineated into $(a) \mathrm{H} \cdots \mathrm{H}$ contacts and (b) $\mathrm{H} \cdots \mathrm{Cl} / \mathrm{Cl} \cdots \mathrm{H}$ contacts for the Te1- and Te2-molecules. The red circles highlight regions distinguishing the two independent molecules.
Fig. $6 a$, occur at shorter distances that those of the Te1-molecules. With regard to the $\mathrm{H} \cdots \mathrm{Cl} / \mathrm{Cl} \cdots \mathrm{H}$ contacts, there is a wider spread at lower $d_{\mathrm{e}}+d_{\mathrm{i}}$ for the Te1- $c f$. the Te2-molecule.

\section{Database survey}

A search of the Cambridge Crystallographic Database (Groom et al., 2016) reveals there are only 28 analogous structures featuring the $\mathrm{TeOC}_{3}$ donor set as in (I) without the bond type being specified. The number of 'hits' reduces to five with the inclusion of the aromatic ring in the side chain. Of the latter, the most closely related compound is 1-bromo-1-butyl3H-2,1-benzoxatellurol (Maksimenko et al., 1994), which is in fact very similar to (I), being derived from this by substituting the tellurium-bound chlorido atom with bromido and the removal of the methyl group. Here, the five-membered chelate ring is strictly planar.

\section{Inhibition of cathepsin B}

Compound (I) was screened for its ability to inhibit cathepsin B employing standard literature procedures (Cunha et al., 2005). The determined value of the inhibition constant was 372 $\pm 40 \mathrm{M}^{-1} \mathrm{~s}^{-1}$, indicating some inhibitory potential, but not as potent as for other organotellurium(IV) compounds studied earlier (Cunha et al., 2005).

\section{Synthesis and crystallization}

The compound was prepared following a literature procedure (Engman, 1984). The precursor chalcogenide, [2- $(R)$ $\mathrm{MeCH}(\mathrm{OH})] \mathrm{C}_{6} \mathrm{H}_{4} \mathrm{Te}(n \mathrm{Bu})(1.52 \mathrm{~g}, 5 \mathrm{mmol})$, prepared as in the literature (Piovan et al., 2011), was dissolved in dry dichloromethane $(20 \mathrm{ml})$ and cooled to $253 \mathrm{~K}$. To the stirred, cooled solution, sulfuryl chloride $(0.4 \mathrm{ml}, 5 \mathrm{mmol})$ dissolved in dichloromethane $(5 \mathrm{ml})$ was added dropwise. The stirring was maintained for 20 minutes at $273 \mathrm{~K}$ and the solvent was then removed under reduced pressure. The oily product thus obtained was purified by crystallization from a mixture of dry benzene and pentane, yielding colourless crystals in $89 \%$ yield, m.p. 641.3-641.4 K. Analysis calculated for $\mathrm{C}_{12} \mathrm{H}_{17} \mathrm{OClTe}$ : C, 42.35, H, 5.03; Found C, 42.28, H, 4.98\%. $[\alpha]_{\mathrm{D}}{ }^{26}=+45.5^{\circ}$ $\left(\mathrm{CHCl}_{3}, c=1.97\right) .{ }^{1} \mathrm{H}\left(500.13 \mathrm{MHz}, \mathrm{CDCl}_{3}, \mathrm{ppm}\right) \delta 8.20\left(d,{ }^{3} \mathrm{~J}\right.$ $7.6 \mathrm{~Hz}, 1 \mathrm{H}), 7.6-7.5(m, 2 \mathrm{H}), 7.31\left(d,{ }^{3} J 7.2 \mathrm{~Hz}, 1 \mathrm{H}\right), 5.59\left(q,{ }^{3} \mathrm{~J}\right.$ $6.3 \mathrm{~Hz}, 1 \mathrm{H}), 3.31\left(t,{ }^{3} J 8.1 \mathrm{~Hz}, 2 \mathrm{H}\right), 1.90\left(\right.$ quin, $\left.{ }^{3} J 7.2 \mathrm{~Hz}, 2 \mathrm{H}\right)$, $1.59\left(d,{ }^{3} J 6.45 \mathrm{~Hz}, 3 \mathrm{H}\right), 1.46\left(\operatorname{sext},{ }^{3} J 7.4 \mathrm{~Hz}, 2 \mathrm{H}\right), 0.93\left(t,{ }^{3} J\right.$ $7.4 \mathrm{~Hz}, 3 \mathrm{H}) .{ }^{13} \mathrm{C}\left(125 \mathrm{MHz}, \mathrm{CDCl}_{3}, \mathrm{ppm}\right) \delta 148.1,131.6,131.2$, 128.7, 127.8, 125.4, $75.5(\mathrm{Br}), 45.4,28.4,24.6,23.7,13.0 .{ }^{125} \mathrm{Te}$ (157.85 MHz, $\left.\mathrm{CDCl}_{3}-d_{6}, \mathrm{ppm}\right) \delta 847.2$ (minor), 801.1 (major). ${ }^{125} \mathrm{Te}\left(157.85 \mathrm{MHz}, \mathrm{DMSO}-d_{6}\right.$, ppm) $\delta 1201.5$ (minor), 1189.1 (major).

\section{Refinement details}

Crystal data, data collection and structure refinement details are summarized in Table 3 . The carbon-bound $\mathrm{H}$-atoms were placed in calculated positions $(\mathrm{C}-\mathrm{H}=0.93-0.98 \AA)$ and were 
included in the refinement in the riding-model approximation, with $U_{\text {iso }}(\mathrm{H})$ set to $1.2-1.5 U_{\text {eq }}(\mathrm{C})$.

\section{Acknowledgements}

The Brazilian agency National Council for Scientific and Technological Development, CNPq, for a scholarship to JZ-S (305626/2013-2).

\section{Funding information}

Funding for this research was provided by: National Council for Scientific and Technological Development (award No. 305626/2013-2).

\section{References}

Albeck, A., Weitman, H., Sredni, B. \& Albeck, M. (1998). Inorg. Chem. 37, 1704-1712.

Brandenburg, K. (2006). DIAMOND. Crystal Impact GbR, Bonn, Germany.

Brodsky, M., Halpert, G., Albeck, M. \& Sredni, B. J. (2010). J. Inflamm. 7, doi 10.1186/1476-9255-7-3.

Burla, M. C., Caliandro, R., Carrozzini, B., Cascarano, G. L., Cuocci, C., Giacovazzo, C., Mallamo, M., Mazzone, A. \& Polidori, G. (2015). J. Appl. Cryst. 48, 306-309.

Caracelli, I., Vega-Teijido, M., Zukerman-Schpector, J., Cezari, M. H. S., Lopes, J. G. S., Juliano, L., Santos, P. S., Comasseto, J. V., Cunha, R. L. O. R. \& Tiekink, E. R. T. (2012). J. Mol. Struct. 1013, 11-18.

Caracelli, I., Zukerman-Schpector, J., Madureira, L. S., Maganhi, S. H., Stefani, H. A., Guadagnin, R. C. \& Tiekink, E. R. T. (2016). Z. Kristallogr. 231, 321-328.

Coppens, P., Leiserowitz, L. \& Rabinovich, D. (1965). Acta Cryst. 18, 1035-1038.

Cunha, R. L. O. R., Urano, M. E., Chagas, J. R., Almeida, P. C., Bincoletto, C., Tersariol, I. L. S. \& Comasseto, J. V. (2005). Bioorg. Med. Chem. Lett. 15, 755-760.

Engman, L. (1984). Organometallics, 3, 1308-1309.

Farrugia, L. J. (2012). J. Appl. Cryst. 45, 849-854.

Fleming, A. (1932). J. Pathol. 35, 831-842.

Friedman, M., Bayer, I., Letko, I., Duvdevani, R., Zavaro-Levy, O., Ron, B., Albeck, M. \& Sredni, B. (2009). Br. J. Dermatol. 160, $403-$ 408.

Gans, J. \& Shalloway, D. (2001). J. Mol. Graphics Modell. 19, 557-559.

Groom, C. R., Bruno, I. J., Lightfoot, M. P. \& Ward, S. C. (2016). Acta Cryst. B72, 171-179.

Halpert, G. \& Sredni, B. (2014). Autoimmun. Rev. 13, 1230-1235.

Jotani, M. M., Zukerman-Schpector, J., Madureira, L. S., Poplaukhin, P., Arman, H. D., Miller, T. \& Tiekink, E. R. T. (2016). Z. Kristallogr. 231, 415-425.

Maksimenko, A. A., Sadekov, I. D., Kompan, O. E., Minkin, V. I. \& Struchkov, Yu. T. (1994). Chem. Heterocycl. Compd. 30, 367-369.

Nogueira, C. W., Zeni, G. W. \& Rocha, J. B. (2004). Chem. Rev. 104, 6255-6286.

Nonius (1998). COLLECT. Nonius BV, Delft, The Netherlands.
Table 3

Experimental details.

\begin{tabular}{|c|c|}
\hline \multicolumn{2}{|l|}{ Crystal data } \\
\hline Chemical formula & $\mathrm{C}_{12} \mathrm{H}_{17} \mathrm{ClOTe}$ \\
\hline$M_{\mathrm{r}}$ & 340.30 \\
\hline Crystal system, space group & Monoclinic, $P 2_{1}$ \\
\hline Temperature $(\mathrm{K})$ & 293 \\
\hline$a, b, c(\AA)$ & $8.3663(2), 13.0442(4), 12.5363(2)$ \\
\hline$\beta\left(^{\circ}\right)$ & $103.460(2)$ \\
\hline$V\left(\AA^{3}\right)$ & $1330.53(6)$ \\
\hline$Z$ & 4 \\
\hline Radiation type & Мо $K \alpha$ \\
\hline$\mu\left(\mathrm{mm}^{-1}\right)$ & 2.41 \\
\hline Crystal size $(\mathrm{mm})$ & $0.34 \times 0.33 \times 0.23$ \\
\hline \multicolumn{2}{|l|}{ Data collection } \\
\hline Diffractometer & Nonius KappaCCD \\
\hline Absorption correction & Gaussian (Coppens et al., 1965) \\
\hline$T_{\min }, T_{\max }$ & $0.481,0.550$ \\
\hline $\begin{array}{l}\text { No. of measured, independent and } \\
\text { observed }[I>2 \sigma(I)] \text { reflections }\end{array}$ & $9220,5115,4998$ \\
\hline$R_{\text {int }}$ & 0.061 \\
\hline$(\sin \theta / \lambda)_{\max }\left(\AA^{-1}\right)$ & 0.650 \\
\hline \multicolumn{2}{|l|}{ Refinement } \\
\hline$R\left[F^{2}>2 \sigma\left(F^{2}\right)\right], w R\left(F^{2}\right), S$ & $0.027,0.076,1.02$ \\
\hline No. of reflections & 5115 \\
\hline No. of parameters & 275 \\
\hline No. of restraints & 1 \\
\hline $\mathrm{H}$-atom treatment & $\mathrm{H}$-atom parameters constrained \\
\hline$\Delta \rho_{\max }, \Delta \rho_{\min }\left(\mathrm{e} \AA^{-3}\right)$ & $0.43,-0.82$ \\
\hline Absolute structure & $\begin{array}{l}\text { Flack } x \text { determined using } 1908 \\
\quad \text { quotients }\left[\left(I^{+}\right)-\left(I^{-}\right)\right] /\left[\left(I^{+}\right)+\left(I^{-}\right)\right] \\
\quad \text { (Parsons } \text { et al., } 2013)\end{array}$ \\
\hline Absolute structure parameter & $-0.05(3)$ \\
\hline
\end{tabular}

Computer programs: COLLECT (Nonius, 1998), DENZO/SCALEPACK (Otwinowski \& Minor, 1997), SIR2014 (Burla et al., 2015), SHELXL2014 (Sheldrick, 2015), ORTEP-3 for Windows (Farrugia, 2012), QMol (Gans \& Shalloway, 2001), DIAMOND (Brandenburg, 2006) and publCIF (Westrip, 2010).

Otwinowski, Z. \& Minor, W. (1997). Methods in Enzymology, Vol. 276, Macromolecular Crystallography, Part A, edited by C. W. Carter Jr \& R. M. Sweet, pp. 307-326. New York: Academic Press. Parsons, S., Flack, H. D. \& Wagner, T. (2013). Acta Cryst. B69, 249 259.

Piovan, L. M. F. M., Alves, M. F. M., Juliano, L., Brömme, D., Cunha, R. L. O. R. \& Andrade, L. H. (2011). Bioorg. Med. Chem. 19, 20092014.

Seng, H. L. \& Tiekink, E. R. T. (2012). Appl. Organomet. Chem. 26, 655-662.

Sheldrick, G. M. (2015). Acta Cryst. C71, 3-8.

Silberman, A., Kalechman, Y., Hirsch, S., Erlich, Z., Sredni, B. \& Albeck, A. (2016). ChemBioChem, 17, 918-927.

Spek, A. L. (2009). Acta Cryst. D65, 148-155.

Sredni, B. (2012). Semin. Cancer Biol. 22, 60-69.

Tiekink, E. R. T. (2012). Dalton Trans. 41, 6390-6395.

Westrip, S. P. (2010). J. Appl. Cryst. 43, 920-925. 


\section{supporting information}

Acta Cryst. (2017). E73, 564-568 [https://doi.org/10.1107/S2056989017003887]

\section{1-Butyl-1-chloro-3-methyl-3H-2,1 $\lambda^{4}$-benzoxatellurole: crystal structure and} Hirshfeld analysis

Julio Zukerman-Schpector, Rodrigo Cunha, Álvaro T. Omori, Lucas Sousa Madureira and Edward R. T. Tiekink

Computing details

Data collection: COLLECT (Nonius, 1998); cell refinement: SCALEPACK (Otwinowski \& Minor, 1997); data reduction: DENZO/SCALEPACK (Otwinowski \& Minor, 1997); program(s) used to solve structure: SIR2014 (Burla et al., 2015); program(s) used to refine structure: SHELXL2014 (Sheldrick, 2015); molecular graphics: ORTEP-3 for Windows (Farrugia, 2012), QMol (Gans \& Shalloway, 2001) and DIAMOND (Brandenburg, 2006); software used to prepare material for publication: publCIF (Westrip, 2010).

1-Butyl-1-chloro-3-methyl-3H-2,1 $\lambda^{4}$-benzoxatellurole

Crystal data

$\mathrm{C}_{12} \mathrm{H}_{17} \mathrm{ClOTe}$

$M_{r}=340.30$

Monoclinic, $P 2_{1}$ $a=8.3663(2) \AA$

$b=13.0442$ (4) $\AA$

$c=12.5363(2) \AA$

$\beta=103.460(2)^{\circ}$

$V=1330.53(6) \AA^{3}$

$Z=4$

$$
F(000)=664
$$

$D_{\mathrm{x}}=1.699 \mathrm{Mg} \mathrm{m}^{-3}$

Mo $K \alpha$ radiation, $\lambda=0.71073 \AA$

Cell parameters from 5903 reflections

$\theta=1.0-27.5^{\circ}$

$\mu=2.41 \mathrm{~mm}^{-1}$

$T=293 \mathrm{~K}$

Slab, colourless

$0.34 \times 0.33 \times 0.23 \mathrm{~mm}$

Data collection

Nonius KappaCCD

5115 independent reflections diffractometer

CCD rotation images, thick slices scans

Absorption correction: gaussian

(Coppens et al., 1965)

$T_{\min }=0.481, T_{\max }=0.550$

9220 measured reflections 4998 reflections with $I>2 \sigma(I)$

$R_{\text {int }}=0.061$

$\theta_{\max }=27.5^{\circ}, \theta_{\min }=2.5^{\circ}$

$h=-10 \rightarrow 8$

$k=-15 \rightarrow 16$

$l=-16 \rightarrow 13$

\section{Refinement}

Refinement on $F^{2}$

Least-squares matrix: full

$R\left[F^{2}>2 \sigma\left(F^{2}\right)\right]=0.027$

$w R\left(F^{2}\right)=0.076$

$S=1.02$

5115 reflections

275 parameters

1 restraint

Hydrogen site location: inferred from neighbouring sites

$\mathrm{H}$-atom parameters constrained

$w=1 /\left[\sigma^{2}\left(F_{\mathrm{o}}^{2}\right)+(0.0389 P)^{2}+0.6419 P\right]$

where $P=\left(F_{\mathrm{o}}{ }^{2}+2 F_{\mathrm{c}}{ }^{2}\right) / 3$

$(\Delta / \sigma)_{\max }<0.001$

$\Delta \rho_{\max }=0.43 \mathrm{e} \AA^{-3}$

$\Delta \rho_{\min }=-0.82$ e $\AA^{-3}$ 
Absolute structure: Flack $x$ determined using

1908 quotients $\left[\left(I^{+}\right)-\left(I^{\prime}\right)\right] /\left[\left(I^{+}\right)+(I)\right]$ (Parsons et al., 2013)

Absolute structure parameter: -0.05 (3)

Special details

Geometry. All esds (except the esd in the dihedral angle between two 1.s. planes) are estimated using the full covariance matrix. The cell esds are taken into account individually in the estimation of esds in distances, angles and torsion angles; correlations between esds in cell parameters are only used when they are defined by crystal symmetry. An approximate (isotropic) treatment of cell esds is used for estimating esds involving l.s. planes.

Fractional atomic coordinates and isotropic or equivalent isotropic displacement parameters $\left(\AA^{2}\right)$

\begin{tabular}{|c|c|c|c|c|}
\hline & $x$ & $y$ & $z$ & $U_{\text {iso }} * / U_{\text {eq }}$ \\
\hline Te1 & $0.47914(4)$ & $0.44547(2)$ & $0.33996(3)$ & $0.04143(11)$ \\
\hline $\mathrm{Cl1}$ & $0.6469(2)$ & $0.30011(15)$ & $0.45946(16)$ & 0.0664 (4) \\
\hline O1 & $0.3777(5)$ & $0.5726(4)$ & $0.2598(3)$ & $0.0512(10)$ \\
\hline $\mathrm{C} 1$ & $0.4636(7)$ & $0.6665(4)$ & $0.2894(6)$ & $0.0475(12)$ \\
\hline H1 & 0.4101 & 0.7045 & 0.3388 & $0.057^{*}$ \\
\hline $\mathrm{C} 2$ & $0.4470(10)$ & $0.7276(6)$ & $0.1836(7)$ & $0.075(2)$ \\
\hline $\mathrm{H} 2 \mathrm{~A}$ & 0.5040 & 0.6927 & 0.1361 & $0.112^{*}$ \\
\hline $\mathrm{H} 2 \mathrm{~B}$ & 0.4936 & 0.7946 & 0.2005 & $0.112 *$ \\
\hline $\mathrm{H} 2 \mathrm{C}$ & 0.3328 & 0.7340 & 0.1476 & $0.112 *$ \\
\hline $\mathrm{C} 3$ & $0.6380(6)$ & $0.6452(5)$ & $0.3476(5)$ & $0.0458(11)$ \\
\hline $\mathrm{C} 4$ & $0.7594(8)$ & $0.7216(6)$ & $0.3717(7)$ & $0.0652(17)$ \\
\hline $\mathrm{H} 4$ & 0.7345 & 0.7880 & 0.3462 & $0.078^{*}$ \\
\hline $\mathrm{C} 5$ & $0.9147(8)$ & $0.6994(8)$ & $0.4326(7)$ & $0.072(2)$ \\
\hline $\mathrm{H} 5$ & 0.9941 & 0.7505 & 0.4491 & $0.086^{*}$ \\
\hline C6 & $0.9523(7)$ & $0.5984(9)$ & $0.4698(6)$ & $0.075(3)$ \\
\hline H6 & 1.0570 & 0.5831 & 0.5113 & $0.090^{*}$ \\
\hline $\mathrm{C} 7$ & $0.8366(6)$ & $0.5227(6)$ & $0.4454(5)$ & $0.0533(15)$ \\
\hline $\mathrm{H} 7$ & 0.8622 & 0.4560 & 0.4695 & $0.064^{*}$ \\
\hline $\mathrm{C} 8$ & $0.6800(6)$ & $0.5467(5)$ & $0.3842(4)$ & $0.0416(10)$ \\
\hline C9 & $0.3754(6)$ & $0.4771(5)$ & $0.4770(4)$ & $0.0474(13)$ \\
\hline H9A & 0.2963 & 0.5322 & 0.4567 & $0.057^{*}$ \\
\hline H9B & 0.3154 & 0.4168 & 0.4909 & $0.057^{*}$ \\
\hline $\mathrm{C} 10$ & $0.4927(6)$ & $0.5063(6)$ & $0.5816(5)$ & $0.0510(14)$ \\
\hline $\mathrm{H} 10 \mathrm{~A}$ & 0.5533 & 0.5668 & 0.5691 & $0.061 *$ \\
\hline H10B & 0.5711 & 0.4511 & 0.6040 & $0.061 *$ \\
\hline C11 & $0.4067(7)$ & $0.5281(5)$ & $0.6735(4)$ & 0.0479 (14) \\
\hline H11A & 0.3329 & 0.5858 & 0.6524 & $0.057^{*}$ \\
\hline H11B & 0.3405 & 0.4691 & 0.6824 & $0.057 *$ \\
\hline $\mathrm{C} 12$ & $0.5220(8)$ & $0.5516(9)$ & $0.7815(5)$ & $0.0674(18)$ \\
\hline $\mathrm{H} 12 \mathrm{~A}$ & 0.6033 & 0.4984 & 0.7992 & $0.101 *$ \\
\hline H12B & 0.4614 & 0.5553 & 0.8376 & $0.101^{*}$ \\
\hline $\mathrm{H} 12 \mathrm{C}$ & 0.5753 & 0.6161 & 0.7767 & $0.101^{*}$ \\
\hline $\mathrm{Te} 2$ & $0.01522(4)$ & $0.55421(2)$ & $0.17723(3)$ & $0.04226(11)$ \\
\hline $\mathrm{Cl} 2$ & $-0.1556(2)$ & $0.69208(15)$ & $0.05090(16)$ & $0.0666(4)$ \\
\hline $\mathrm{O} 2$ & $0.1199(5)$ & 0.4303 (4) & $0.2604(4)$ & $0.0583(11)$ \\
\hline
\end{tabular}




$\begin{array}{lllll}\text { C13 } & 0.0191(7) & 0.3453(5) & 0.2705(5) & 0.0530(13) \\ \text { H13 } & 0.0032 & 0.3452 & 0.3455 & 0.064^{*} \\ \text { C14 } & 0.1031(10) & 0.2471(6) & 0.2538(9) & 0.083(3) \\ \text { H14A } & 0.1177 & 0.2445 & 0.1801 & 0.125^{*} \\ \text { H14B } & 0.0371 & 0.1901 & 0.2661 & 0.125^{*} \\ \text { H14C } & 0.2085 & 0.2439 & 0.3045 & 0.125^{*} \\ \text { C15 } & -0.1500(7) & 0.3589(5) & 0.1920(5) & 0.0498(13) \\ \text { C16 } & -0.2697(8) & 0.2822(6) & 0.1731(6) & 0.0627(16) \\ \text { H16 } & -0.2486 & 0.2191 & 0.2080 & 0.075^{*} \\ \text { C17 } & -0.4201(8) & 0.3008(7) & 0.1021(6) & 0.0646(19) \\ \text { H17 } & -0.4989 & 0.2492 & 0.0881 & 0.077^{*} \\ \text { C18 } & -0.4547(7) & 0.3946(7) & 0.0519(5) & 0.0585(17) \\ \text { H18 } & -0.5568 & 0.4059 & 0.0048 & 0.070^{*} \\ \text { C19 } & -0.3397(7) & 0.4717(5) & 0.0709(5) & 0.0508(14) \\ \text { H19 } & -0.3636 & 0.5356 & 0.0380 & 0.061^{*} \\ \text { C20 } & -0.1858(5) & 0.4524(5) & 0.1407(4) & 0.0413(10) \\ \text { C21 } & 0.1184(6) & 0.5194(6) & 0.0408(5) & 0.0546(16) \\ \text { H21A } & 0.1644 & 0.5819 & 0.0185 & 0.066^{*} \\ \text { H21B } & 0.2084 & 0.4717 & 0.0652 & 0.066^{*} \\ \text { C22 } & 0.0033(7) & 0.4747(5) & -0.0574(5) & 0.0485(14) \\ \text { H22A } & -0.0914 & 0.5194 & -0.0794 & 0.058^{*} \\ \text { H22B } & -0.0352 & 0.4088 & -0.0380 & 0.058^{*} \\ \text { C23 } & 0.0837(7) & 0.4607(6) & -0.1542(5) & 0.0506(13) \\ \text { H23A } & 0.1271 & 0.5262 & -0.1710 & 0.061^{*} \\ \text { H23B } & 0.1753 & 0.4137 & -0.1330 & 0.061^{*} \\ \text { C24 } & -0.0311(9) & 0.4209(8) & -0.2549(6) & 0.079(3) \\ \text { H24A } & -0.0737 & 0.3556 & -0.2392 & 0.118^{*} \\ \text { H24B } & 0.0266 & 0.4130 & -0.3122 & 0.118^{*} \\ \text { H24C } & -0.1203 & 0.4682 & -0.2780 & 0.118^{*} \\ & & & & \end{array}$

Atomic displacement parameters $\left(\AA^{2}\right)$

\begin{tabular}{lllllll}
\hline & $U^{11}$ & $U^{22}$ & $U^{33}$ & $U^{12}$ & $U^{13}$ & $U^{23}$ \\
\hline Te1 & $0.04719(18)$ & $0.0443(2)$ & $0.02938(15)$ & $0.00273(13)$ & $0.00198(11)$ & $-0.00116(15)$ \\
C11 & $0.0837(11)$ & $0.0546(9)$ & $0.0580(10)$ & $0.0195(8)$ & $0.0106(8)$ & $0.0102(7)$ \\
O1 & $0.0467(18)$ & $0.050(3)$ & $0.047(2)$ & $0.0016(17)$ & $-0.0085(15)$ & $0.0090(18)$ \\
C1 & $0.052(3)$ & $0.033(2)$ & $0.055(3)$ & $0.004(2)$ & $0.007(2)$ & $-0.003(3)$ \\
C2 & $0.079(4)$ & $0.060(4)$ & $0.076(5)$ & $0.000(3)$ & $0.000(4)$ & $0.025(4)$ \\
C3 & $0.047(2)$ & $0.049(3)$ & $0.042(3)$ & $-0.001(2)$ & $0.012(2)$ & $-0.003(2)$ \\
C4 & $0.065(4)$ & $0.059(4)$ & $0.070(5)$ & $-0.014(3)$ & $0.012(3)$ & $-0.002(3)$ \\
C5 & $0.046(3)$ & $0.099(6)$ & $0.068(5)$ & $-0.022(3)$ & $0.009(3)$ & $-0.004(4)$ \\
C6 & $0.034(3)$ & $0.133(8)$ & $0.054(4)$ & $0.001(4)$ & $0.005(2)$ & $-0.014(5)$ \\
C7 & $0.039(2)$ & $0.078(5)$ & $0.042(3)$ & $0.010(2)$ & $0.008(2)$ & $0.001(3)$ \\
C8 & $0.041(2)$ & $0.055(3)$ & $0.030(2)$ & $0.003(2)$ & $0.0091(17)$ & $0.000(2)$ \\
C9 & $0.040(2)$ & $0.067(4)$ & $0.034(2)$ & $-0.001(2)$ & $0.0074(19)$ & $-0.004(2)$ \\
C10 & $0.039(2)$ & $0.077(4)$ & $0.037(3)$ & $0.000(2)$ & $0.008(2)$ & $-0.010(3)$ \\
C11 & $0.047(2)$ & $0.061(4)$ & $0.036(3)$ & $0.002(2)$ & $0.010(2)$ & $-0.002(2)$ \\
C12 & $0.062(3)$ & $0.096(5)$ & $0.043(3)$ & $0.002(4)$ & $0.008(2)$ & $-0.022(4)$
\end{tabular}




\begin{tabular}{lllllll}
$\mathrm{Te} 2$ & $0.04777(18)$ & $0.0432(2)$ & $0.03135(16)$ & $0.00212(13)$ & $0.00019(12)$ & $-0.00352(14)$ \\
$\mathrm{C} 12$ & $0.0768(10)$ & $0.0535(9)$ & $0.0646(10)$ & $0.0164(8)$ & $0.0063(8)$ & $0.0099(8)$ \\
$\mathrm{O} 2$ & $0.052(2)$ & $0.056(3)$ & $0.055(2)$ & $-0.001(2)$ & $-0.0130(17)$ & $0.008(2)$ \\
$\mathrm{C} 13$ & $0.060(3)$ & $0.056(3)$ & $0.037(3)$ & $0.001(3)$ & $-0.001(2)$ & $0.001(3)$ \\
$\mathrm{C} 14$ & $0.073(4)$ & $0.058(4)$ & $0.110(7)$ & $0.012(3)$ & $0.003(4)$ & $-0.004(4)$ \\
$\mathrm{C} 15$ & $0.052(3)$ & $0.054(3)$ & $0.041(3)$ & $-0.005(2)$ & $0.005(2)$ & $0.001(2)$ \\
$\mathrm{C} 16$ & $0.063(3)$ & $0.065(4)$ & $0.059(4)$ & $-0.009(3)$ & $0.013(3)$ & $0.002(3)$ \\
$\mathrm{C} 17$ & $0.055(3)$ & $0.081(5)$ & $0.059(4)$ & $-0.021(3)$ & $0.013(3)$ & $-0.017(4)$ \\
$\mathrm{C} 18$ & $0.038(3)$ & $0.087(5)$ & $0.050(3)$ & $-0.003(3)$ & $0.009(2)$ & $-0.014(3)$ \\
$\mathrm{C} 19$ & $0.047(3)$ & $0.067(4)$ & $0.037(3)$ & $0.010(2)$ & $0.006(2)$ & $0.000(2)$ \\
$\mathrm{C} 20$ & $0.040(2)$ & $0.054(3)$ & $0.029(2)$ & $0.002(2)$ & $0.0053(16)$ & $-0.006(2)$ \\
$\mathrm{C} 21$ & $0.042(2)$ & $0.083(5)$ & $0.037(3)$ & $-0.001(3)$ & $0.005(2)$ & $-0.010(3)$ \\
$\mathrm{C} 22$ & $0.046(2)$ & $0.061(4)$ & $0.039(3)$ & $0.003(2)$ & $0.010(2)$ & $-0.008(2)$ \\
$\mathrm{C} 23$ & $0.049(2)$ & $0.062(4)$ & $0.041(3)$ & $0.005(2)$ & $0.012(2)$ & $0.001(3)$ \\
$\mathrm{C} 24$ & $0.068(4)$ & $0.121(9)$ & $0.045(3)$ & $0.015(4)$ & $0.008(3)$ & $-0.019(4)$ \\
& & & & & & \\
\hline
\end{tabular}

Geometric parameters $\left(\AA,{ }^{\circ}\right)$

\begin{tabular}{|c|c|c|c|}
\hline $\mathrm{Te} 1-\mathrm{Cl1}$ & $2.6137(17)$ & C11-H11A & 0.9700 \\
\hline $\mathrm{Te} 1-\mathrm{O} 1$ & $2.021(4)$ & C11-H11B & 0.9700 \\
\hline $\mathrm{Te} 1-\mathrm{C} 8$ & $2.107(6)$ & $\mathrm{C} 12-\mathrm{H} 12 \mathrm{~A}$ & 0.9600 \\
\hline $\mathrm{Te} 1-\mathrm{C} 9$ & $2.138(5)$ & $\mathrm{C} 12-\mathrm{H} 12 \mathrm{~B}$ & 0.9600 \\
\hline $\mathrm{Te} 1-\mathrm{O} 2$ & $2.945(4)$ & $\mathrm{C} 12-\mathrm{H} 12 \mathrm{C}$ & 0.9600 \\
\hline $\mathrm{Te} 2-\mathrm{Cl} 2$ & $2.5944(17)$ & $\mathrm{O} 2-\mathrm{C} 13$ & $1.416(8)$ \\
\hline $\mathrm{Te} 2-\mathrm{O} 2$ & $2.010(5)$ & $\mathrm{C} 13-\mathrm{C} 14$ & $1.499(10)$ \\
\hline $\mathrm{Te} 2-\mathrm{C} 20$ & $2.108(6)$ & $\mathrm{C} 13-\mathrm{C} 15$ & $1.534(7)$ \\
\hline $\mathrm{Te} 2-\mathrm{C} 21$ & $2.136(6)$ & $\mathrm{C} 13-\mathrm{H} 13$ & 0.9800 \\
\hline $\mathrm{Te} 2-\mathrm{O} 1$ & $2.977(4)$ & $\mathrm{C} 14-\mathrm{H} 14 \mathrm{~A}$ & 0.9600 \\
\hline $\mathrm{O} 1-\mathrm{C} 1$ & $1.424(7)$ & C14-H14B & 0.9600 \\
\hline $\mathrm{C} 1-\mathrm{C} 3$ & $1.497(7)$ & $\mathrm{C} 14-\mathrm{H} 14 \mathrm{C}$ & 0.9600 \\
\hline $\mathrm{C} 1-\mathrm{C} 2$ & $1.526(10)$ & $\mathrm{C} 15-\mathrm{C} 20$ & $1.379(9)$ \\
\hline $\mathrm{C} 1-\mathrm{H} 1$ & 0.9800 & $\mathrm{C} 15-\mathrm{C} 16$ & $1.396(9)$ \\
\hline $\mathrm{C} 2-\mathrm{H} 2 \mathrm{~A}$ & 0.9600 & $\mathrm{C} 16-\mathrm{C} 17$ & $1.383(9)$ \\
\hline $\mathrm{C} 2-\mathrm{H} 2 \mathrm{~B}$ & 0.9600 & $\mathrm{C} 16-\mathrm{H} 16$ & 0.9300 \\
\hline $\mathrm{C} 2-\mathrm{H} 2 \mathrm{C}$ & 0.9600 & $\mathrm{C} 17-\mathrm{C} 18$ & $1.376(12)$ \\
\hline $\mathrm{C} 3-\mathrm{C} 8$ & $1.382(9)$ & C17-H17 & 0.9300 \\
\hline $\mathrm{C} 3-\mathrm{C} 4$ & $1.405(9)$ & $\mathrm{C} 18-\mathrm{C} 19$ & $1.374(10)$ \\
\hline $\mathrm{C} 4-\mathrm{C} 5$ & $1.376(10)$ & $\mathrm{C} 18-\mathrm{H} 18$ & 0.9300 \\
\hline $\mathrm{C} 4-\mathrm{H} 4$ & 0.9300 & $\mathrm{C} 19-\mathrm{C} 20$ & $1.401(7)$ \\
\hline $\mathrm{C} 5-\mathrm{C} 6$ & $1.408(14)$ & C19-H19 & 0.9300 \\
\hline $\mathrm{C} 5-\mathrm{H} 5$ & 0.9300 & $\mathrm{C} 21-\mathrm{C} 22$ & $1.494(7)$ \\
\hline C6- 77 & $1.367(11)$ & $\mathrm{C} 21-\mathrm{H} 21 \mathrm{~A}$ & 0.9700 \\
\hline C6- $-\mathrm{H} 6$ & 0.9300 & $\mathrm{C} 21-\mathrm{H} 21 \mathrm{~B}$ & 0.9700 \\
\hline $\mathrm{C} 7-\mathrm{C} 8$ & $1.391(7)$ & $\mathrm{C} 22-\mathrm{C} 23$ & $1.529(7)$ \\
\hline $\mathrm{C} 7-\mathrm{H} 7$ & 0.9300 & $\mathrm{C} 22-\mathrm{H} 22 \mathrm{~A}$ & 0.9700 \\
\hline $\mathrm{C} 9-\mathrm{C} 10$ & $1.493(7)$ & $\mathrm{C} 22-\mathrm{H} 22 \mathrm{~B}$ & 0.9700 \\
\hline C9-H9A & 0.9700 & $\mathrm{C} 23-\mathrm{C} 24$ & $1.490(9)$ \\
\hline C9-H9B & 0.9700 & $\mathrm{C} 23-\mathrm{H} 23 \mathrm{~A}$ & 0.9700 \\
\hline
\end{tabular}




\begin{tabular}{|c|c|c|c|}
\hline $\mathrm{C} 10-\mathrm{C} 11$ & $1.521(7)$ & $\mathrm{C} 23-\mathrm{H} 23 \mathrm{~B}$ & 0.9700 \\
\hline $\mathrm{C} 10-\mathrm{H} 10 \mathrm{~A}$ & 0.9700 & $\mathrm{C} 24-\mathrm{H} 24 \mathrm{~A}$ & 0.9600 \\
\hline $\mathrm{C} 10-\mathrm{H} 10 \mathrm{~B}$ & 0.9700 & $\mathrm{C} 24-\mathrm{H} 24 \mathrm{~B}$ & 0.9600 \\
\hline $\mathrm{C} 11-\mathrm{C} 12$ & $1.500(8)$ & $\mathrm{C} 24-\mathrm{H} 24 \mathrm{C}$ & 0.9600 \\
\hline $\mathrm{C} 11-\mathrm{Te} 1-\mathrm{O} 1$ & $171.04(13)$ & $\mathrm{C} 10-\mathrm{C} 11-\mathrm{H} 11 \mathrm{~A}$ & 108.8 \\
\hline $\mathrm{O} 1-\mathrm{Te} 1-\mathrm{C} 8$ & $80.4(2)$ & $\mathrm{C} 12-\mathrm{C} 11-\mathrm{H} 11 \mathrm{~B}$ & 108.8 \\
\hline $\mathrm{C} 8-\mathrm{Te} 1-\mathrm{O} 2$ & $145.0(2)$ & $\mathrm{C} 10-\mathrm{C} 11-\mathrm{H} 11 \mathrm{~B}$ & 108.8 \\
\hline $\mathrm{C} 12-\mathrm{Te} 2-\mathrm{O} 2$ & $170.22(14)$ & $\mathrm{H} 11 \mathrm{~A}-\mathrm{C} 11-\mathrm{H} 11 \mathrm{~B}$ & 107.7 \\
\hline $\mathrm{O} 2-\mathrm{Te} 2-\mathrm{C} 20$ & $80.5(2)$ & $\mathrm{C} 11-\mathrm{C} 12-\mathrm{H} 12 \mathrm{~A}$ & 109.5 \\
\hline $\mathrm{C} 20-\mathrm{Te} 2-\mathrm{O} 1$ & $145.38(19)$ & $\mathrm{C} 11-\mathrm{C} 12-\mathrm{H} 12 \mathrm{~B}$ & 109.5 \\
\hline $\mathrm{O} 1-\mathrm{Te} 1-\mathrm{O} 2$ & $66.99(14)$ & $\mathrm{H} 12 \mathrm{~A}-\mathrm{C} 12-\mathrm{H} 12 \mathrm{~B}$ & 109.5 \\
\hline $\mathrm{O} 1-\mathrm{Te} 1-\mathrm{C} 9$ & $92.2(2)$ & $\mathrm{C} 11-\mathrm{C} 12-\mathrm{H} 12 \mathrm{C}$ & 109.5 \\
\hline $\mathrm{C} 8-\mathrm{Te} 1-\mathrm{C} 9$ & $96.7(2)$ & $\mathrm{H} 12 \mathrm{~A}-\mathrm{C} 12-\mathrm{H} 12 \mathrm{C}$ & 109.5 \\
\hline $\mathrm{C} 8-\mathrm{Te} 1-\mathrm{Cl} 1$ & $90.85(17)$ & $\mathrm{H} 12 \mathrm{~B}-\mathrm{C} 12-\mathrm{H} 12 \mathrm{C}$ & 109.5 \\
\hline C9-Te1-Cl1 & $86.78(17)$ & $\mathrm{C} 13-\mathrm{O} 2-\mathrm{Te} 2$ & $118.6(3)$ \\
\hline $\mathrm{C} 9-\mathrm{Te} 1-\mathrm{O} 2$ & $73.25(17)$ & $\mathrm{C} 13-\mathrm{O} 2-\mathrm{Te} 1$ & $127.2(3)$ \\
\hline $\mathrm{C} 11-\mathrm{Te} 1-\mathrm{O} 2$ & $121.01(11)$ & $\mathrm{Te} 2-\mathrm{O} 2-\mathrm{Te} 1$ & $114.1(2)$ \\
\hline $\mathrm{C} 1-\mathrm{O} 1-\mathrm{Te} 1$ & $116.6(3)$ & $\mathrm{O} 2-\mathrm{C} 13-\mathrm{C} 14$ & $110.4(5)$ \\
\hline $\mathrm{C} 1-\mathrm{O} 1-\mathrm{Te} 2$ & $124.9(3)$ & $\mathrm{O} 2-\mathrm{C} 13-\mathrm{C} 15$ & $109.4(5)$ \\
\hline $\mathrm{Te} 1-\mathrm{O} 1-\mathrm{Te} 2$ & $112.50(18)$ & $\mathrm{C} 14-\mathrm{C} 13-\mathrm{C} 15$ & $113.6(6)$ \\
\hline $\mathrm{O} 2-\mathrm{Te} 2-\mathrm{C} 21$ & $92.1(2)$ & $\mathrm{O} 2-\mathrm{C} 13-\mathrm{H} 13$ & 107.7 \\
\hline $\mathrm{C} 20-\mathrm{Te} 2-\mathrm{C} 21$ & $98.2(2)$ & $\mathrm{C} 14-\mathrm{C} 13-\mathrm{H} 13$ & 107.7 \\
\hline $\mathrm{C} 20-\mathrm{Te} 2-\mathrm{C} 12$ & $90.34(16)$ & $\mathrm{C} 15-\mathrm{C} 13-\mathrm{H} 13$ & 107.7 \\
\hline $\mathrm{C} 21-\mathrm{Te} 2-\mathrm{Cl} 2$ & 85.79 (19) & $\mathrm{C} 13-\mathrm{C} 14-\mathrm{H} 14 \mathrm{~A}$ & 109.5 \\
\hline $\mathrm{O} 2-\mathrm{Te} 2-\mathrm{O} 1$ & $66.36(14)$ & $\mathrm{C} 13-\mathrm{C} 14-\mathrm{H} 14 \mathrm{~B}$ & 109.5 \\
\hline $\mathrm{C} 21-\mathrm{Te} 2-\mathrm{O} 1$ & $74.15(17)$ & $\mathrm{H} 14 \mathrm{~A}-\mathrm{C} 14-\mathrm{H} 14 \mathrm{~B}$ & 109.5 \\
\hline $\mathrm{C} 12-\mathrm{Te} 2-\mathrm{O} 1$ & $121.88(10)$ & $\mathrm{C} 13-\mathrm{C} 14-\mathrm{H} 14 \mathrm{C}$ & 109.5 \\
\hline $\mathrm{O} 1-\mathrm{C} 1-\mathrm{C} 3$ & $110.1(5)$ & $\mathrm{H} 14 \mathrm{~A}-\mathrm{C} 14-\mathrm{H} 14 \mathrm{C}$ & 109.5 \\
\hline $\mathrm{O} 1-\mathrm{C} 1-\mathrm{C} 2$ & $106.5(6)$ & $\mathrm{H} 14 \mathrm{~B}-\mathrm{C} 14-\mathrm{H} 14 \mathrm{C}$ & 109.5 \\
\hline $\mathrm{C} 3-\mathrm{C} 1-\mathrm{C} 2$ & $113.7(5)$ & $\mathrm{C} 20-\mathrm{C} 15-\mathrm{C} 16$ & $119.0(5)$ \\
\hline $\mathrm{O} 1-\mathrm{C} 1-\mathrm{H} 1$ & 108.8 & $\mathrm{C} 20-\mathrm{C} 15-\mathrm{C} 13$ & $118.0(5)$ \\
\hline $\mathrm{C} 3-\mathrm{C} 1-\mathrm{H} 1$ & 108.8 & $\mathrm{C} 16-\mathrm{C} 15-\mathrm{C} 13$ & $123.0(6)$ \\
\hline $\mathrm{C} 2-\mathrm{C} 1-\mathrm{H} 1$ & 108.8 & $\mathrm{C} 17-\mathrm{C} 16-\mathrm{C} 15$ & $119.5(7)$ \\
\hline $\mathrm{C} 1-\mathrm{C} 2-\mathrm{H} 2 \mathrm{~A}$ & 109.5 & $\mathrm{C} 17-\mathrm{C} 16-\mathrm{H} 16$ & 120.3 \\
\hline $\mathrm{C} 1-\mathrm{C} 2-\mathrm{H} 2 \mathrm{~B}$ & 109.5 & $\mathrm{C} 15-\mathrm{C} 16-\mathrm{H} 16$ & 120.3 \\
\hline $\mathrm{H} 2 \mathrm{~A}-\mathrm{C} 2-\mathrm{H} 2 \mathrm{~B}$ & 109.5 & $\mathrm{C} 16-\mathrm{C} 17-\mathrm{C} 18$ & $120.9(7)$ \\
\hline $\mathrm{C} 1-\mathrm{C} 2-\mathrm{H} 2 \mathrm{C}$ & 109.5 & $\mathrm{C} 16-\mathrm{C} 17-\mathrm{H} 17$ & 119.5 \\
\hline $\mathrm{H} 2 \mathrm{~A}-\mathrm{C} 2-\mathrm{H} 2 \mathrm{C}$ & 109.5 & $\mathrm{C} 18-\mathrm{C} 17-\mathrm{H} 17$ & 119.5 \\
\hline $\mathrm{H} 2 \mathrm{~B}-\mathrm{C} 2-\mathrm{H} 2 \mathrm{C}$ & 109.5 & $\mathrm{C} 19-\mathrm{C} 18-\mathrm{C} 17$ & $120.5(6)$ \\
\hline $\mathrm{C} 8-\mathrm{C} 3-\mathrm{C} 4$ & $118.3(5)$ & $\mathrm{C} 19-\mathrm{C} 18-\mathrm{H} 18$ & 119.8 \\
\hline $\mathrm{C} 8-\mathrm{C} 3-\mathrm{C} 1$ & $118.5(5)$ & $\mathrm{C} 17-\mathrm{C} 18-\mathrm{H} 18$ & 119.8 \\
\hline $\mathrm{C} 4-\mathrm{C} 3-\mathrm{C} 1$ & $123.1(6)$ & $\mathrm{C} 18-\mathrm{C} 19-\mathrm{C} 20$ & $118.7(6)$ \\
\hline $\mathrm{C} 5-\mathrm{C} 4-\mathrm{C} 3$ & $120.8(8)$ & $\mathrm{C} 18-\mathrm{C} 19-\mathrm{H} 19$ & 120.6 \\
\hline $\mathrm{C} 5-\mathrm{C} 4-\mathrm{H} 4$ & 119.6 & $\mathrm{C} 20-\mathrm{C} 19-\mathrm{H} 19$ & 120.6 \\
\hline $\mathrm{C} 3-\mathrm{C} 4-\mathrm{H} 4$ & 119.6 & $\mathrm{C} 15-\mathrm{C} 20-\mathrm{C} 19$ & $121.3(6)$ \\
\hline $\mathrm{C} 4-\mathrm{C} 5-\mathrm{C} 6$ & $119.4(7)$ & $\mathrm{C} 15-\mathrm{C} 20-\mathrm{Te} 2$ & $112.3(3)$ \\
\hline $\mathrm{C} 4-\mathrm{C} 5-\mathrm{H} 5$ & 120.3 & $\mathrm{C} 19-\mathrm{C} 20-\mathrm{Te} 2$ & $126.4(5)$ \\
\hline
\end{tabular}




\begin{tabular}{|c|c|}
\hline $\mathrm{C} 6-\mathrm{C} 5-\mathrm{H} 5$ & 120.3 \\
\hline $\mathrm{C} 7-\mathrm{C} 6-\mathrm{C} 5$ & $120.6(6)$ \\
\hline $\mathrm{C} 7-\mathrm{C} 6-\mathrm{H} 6$ & 119.7 \\
\hline $\mathrm{C} 5-\mathrm{C} 6-\mathrm{H} 6$ & 119.7 \\
\hline $\mathrm{C} 6-\mathrm{C} 7-\mathrm{C} 8$ & $119.2(7)$ \\
\hline $\mathrm{C} 6-\mathrm{C} 7-\mathrm{H} 7$ & 120.4 \\
\hline $\mathrm{C} 8-\mathrm{C} 7-\mathrm{H} 7$ & 120.4 \\
\hline $\mathrm{C} 3-\mathrm{C} 8-\mathrm{C} 7$ & $121.7(6)$ \\
\hline $\mathrm{C} 3-\mathrm{C} 8-\mathrm{Te} 1$ & $111.7(4)$ \\
\hline $\mathrm{C} 7-\mathrm{C} 8-\mathrm{Te} 1$ & $126.5(5)$ \\
\hline $\mathrm{C} 10-\mathrm{C} 9-\mathrm{Te} 1$ & $116.6(3)$ \\
\hline $\mathrm{C} 10-\mathrm{C} 9-\mathrm{H} 9 \mathrm{~A}$ & 108.1 \\
\hline Te1-C9-H9A & 108.1 \\
\hline $\mathrm{C} 10-\mathrm{C} 9-\mathrm{H} 9 \mathrm{~B}$ & 108.1 \\
\hline Тe1-C9-H9B & 108.1 \\
\hline $\mathrm{H} 9 \mathrm{~A}-\mathrm{C} 9-\mathrm{H} 9 \mathrm{~B}$ & 107.3 \\
\hline $\mathrm{C} 9-\mathrm{C} 10-\mathrm{C} 11$ & $112.5(4)$ \\
\hline $\mathrm{C} 9-\mathrm{C} 10-\mathrm{H} 10 \mathrm{~A}$ & 109.1 \\
\hline $\mathrm{C} 11-\mathrm{C} 10-\mathrm{H} 10 \mathrm{~A}$ & 109.1 \\
\hline $\mathrm{C} 9-\mathrm{C} 10-\mathrm{H} 10 \mathrm{~B}$ & 109.1 \\
\hline $\mathrm{C} 11-\mathrm{C} 10-\mathrm{H} 10 \mathrm{~B}$ & 109.1 \\
\hline $\mathrm{H} 10 \mathrm{~A}-\mathrm{C} 10-\mathrm{H} 10 \mathrm{~B}$ & 107.8 \\
\hline $\mathrm{C} 12-\mathrm{C} 11-\mathrm{C} 10$ & $113.8(5)$ \\
\hline $\mathrm{C} 12-\mathrm{C} 11-\mathrm{H} 11 \mathrm{~A}$ & 108.8 \\
\hline $\mathrm{Te} 1-\mathrm{O} 1-\mathrm{C} 1-\mathrm{C} 3$ & $18.3(7)$ \\
\hline $\mathrm{Te} 2-\mathrm{O} 1-\mathrm{C} 1-\mathrm{C} 3$ & $168.9(3)$ \\
\hline $\mathrm{Te} 1-\mathrm{O} 1-\mathrm{C} 1-\mathrm{C} 2$ & $142.1(4)$ \\
\hline $\mathrm{Te} 2-\mathrm{O} 1-\mathrm{C} 1-\mathrm{C} 2$ & $-67.4(6)$ \\
\hline $\mathrm{O} 1-\mathrm{C} 1-\mathrm{C} 3-\mathrm{C} 8$ & $-13.3(8)$ \\
\hline $\mathrm{C} 2-\mathrm{C} 1-\mathrm{C} 3-\mathrm{C} 8$ & $-132.8(6)$ \\
\hline $\mathrm{O} 1-\mathrm{C} 1-\mathrm{C} 3-\mathrm{C} 4$ & $169.8(6)$ \\
\hline $\mathrm{C} 2-\mathrm{C} 1-\mathrm{C} 3-\mathrm{C} 4$ & $50.4(9)$ \\
\hline $\mathrm{C} 8-\mathrm{C} 3-\mathrm{C} 4-\mathrm{C} 5$ & $-1.8(10)$ \\
\hline $\mathrm{C} 1-\mathrm{C} 3-\mathrm{C} 4-\mathrm{C} 5$ & $175.1(7)$ \\
\hline $\mathrm{C} 3-\mathrm{C} 4-\mathrm{C} 5-\mathrm{C} 6$ & $0.9(12)$ \\
\hline $\mathrm{C} 4-\mathrm{C} 5-\mathrm{C} 6-\mathrm{C} 7$ & $0.3(12)$ \\
\hline $\mathrm{C} 5-\mathrm{C} 6-\mathrm{C} 7-\mathrm{C} 8$ & $-0.6(10)$ \\
\hline $\mathrm{C} 4-\mathrm{C} 3-\mathrm{C} 8-\mathrm{C} 7$ & $1.5(9)$ \\
\hline $\mathrm{C} 1-\mathrm{C} 3-\mathrm{C} 8-\mathrm{C} 7$ & $-175.5(5)$ \\
\hline $\mathrm{C} 4-\mathrm{C} 3-\mathrm{C} 8-\mathrm{Te} 1$ & $179.6(5)$ \\
\hline $\mathrm{C} 1-\mathrm{C} 3-\mathrm{C} 8-\mathrm{Te} 1$ & $2.6(6)$ \\
\hline $\mathrm{C} 6-\mathrm{C} 7-\mathrm{C} 8-\mathrm{C} 3$ & $-0.3(9)$ \\
\hline $\mathrm{C} 6-\mathrm{C} 7-\mathrm{C} 8-\mathrm{Te} 1$ & $-178.1(5)$ \\
\hline $\mathrm{Te} 1-\mathrm{C} 9-\mathrm{C} 10-\mathrm{C} 11$ & $179.5(5)$ \\
\hline $\mathrm{C} 9-\mathrm{C} 10-\mathrm{C} 11-\mathrm{C} 12$ & $176.6(8)$ \\
\hline
\end{tabular}

$\mathrm{C} 22-\mathrm{C} 21-\mathrm{Te} 2 \quad 116.1$ (4)

$\mathrm{C} 22-\mathrm{C} 21-\mathrm{H} 21 \mathrm{~A} \quad 108.3$

$\mathrm{Te} 2-\mathrm{C} 21-\mathrm{H} 21 \mathrm{~A} \quad 108.3$

$\mathrm{C} 22-\mathrm{C} 21-\mathrm{H} 21 \mathrm{~B} \quad 108.3$

$\mathrm{Te} 2-\mathrm{C} 21-\mathrm{H} 21 \mathrm{~B}$

$\mathrm{H} 21 \mathrm{~A}-\mathrm{C} 21-\mathrm{H} 21 \mathrm{~B} \quad 107.4$

$\mathrm{C} 21-\mathrm{C} 22-\mathrm{C} 23 \quad 112.5(5)$

$\mathrm{C} 21-\mathrm{C} 22-\mathrm{H} 22 \mathrm{~A} \quad 109.1$

$\mathrm{C} 23-\mathrm{C} 22-\mathrm{H} 22 \mathrm{~A} \quad 109.1$

$\mathrm{C} 21-\mathrm{C} 22-\mathrm{H} 22 \mathrm{~B} \quad 109.1$

$\mathrm{C} 23-\mathrm{C} 22-\mathrm{H} 22 \mathrm{~B} \quad 109.1$

$\mathrm{H} 22 \mathrm{~A}-\mathrm{C} 22-\mathrm{H} 22 \mathrm{~B} \quad 107.8$

$\mathrm{C} 24-\mathrm{C} 23-\mathrm{C} 22$

$\mathrm{C} 24-\mathrm{C} 23-\mathrm{H} 23 \mathrm{~A} \quad 108.9$

$\mathrm{C} 22-\mathrm{C} 23-\mathrm{H} 23 \mathrm{~A} \quad 108.9$

$\mathrm{C} 24-\mathrm{C} 23-\mathrm{H} 23 \mathrm{~B} \quad 108.9$

$\mathrm{C} 22-\mathrm{C} 23-\mathrm{H} 23 \mathrm{~B} \quad 108.9$

$\mathrm{H} 23 \mathrm{~A}-\mathrm{C} 23-\mathrm{H} 23 \mathrm{~B} \quad 107.7$

$\mathrm{C} 23-\mathrm{C} 24-\mathrm{H} 24 \mathrm{~A}$

$\mathrm{C} 23-\mathrm{C} 24-\mathrm{H} 24 \mathrm{~B} \quad 109.5$

$\mathrm{H} 24 \mathrm{~A}-\mathrm{C} 24-\mathrm{H} 24 \mathrm{~B} \quad 109.5$

$\mathrm{C} 23-\mathrm{C} 24-\mathrm{H} 24 \mathrm{C} \quad 109.5$

$\mathrm{H} 24 \mathrm{~A}-\mathrm{C} 24-\mathrm{H} 24 \mathrm{C} \quad 109.5$

$\mathrm{H} 24 \mathrm{~B}-\mathrm{C} 24-\mathrm{H} 24 \mathrm{C} \quad 109.5$

$\mathrm{Te} 2-\mathrm{O} 2-\mathrm{C} 13-\mathrm{C} 14 \quad 137.8(6)$

$\mathrm{Te} 1-\mathrm{O} 2-\mathrm{C} 13-\mathrm{C} 14 \quad-39.0(7)$

$\mathrm{Te} 2-\mathrm{O} 2-\mathrm{C} 13-\mathrm{C} 15 \quad 12.1(7)$

$\mathrm{Te} 1-\mathrm{O} 2-\mathrm{C} 13-\mathrm{C} 15 \quad-164.8(4)$

$\mathrm{O} 2-\mathrm{C} 13-\mathrm{C} 15-\mathrm{C} 20 \quad-10.0(8)$

$\mathrm{C} 14-\mathrm{C} 13-\mathrm{C} 15-\mathrm{C} 20 \quad-133.9(7)$

$\mathrm{O} 2-\mathrm{C} 13-\mathrm{C} 15-\mathrm{C} 16 \quad 171.9(6)$

$\mathrm{C} 14-\mathrm{C} 13-\mathrm{C} 15-\mathrm{C} 16 \quad 48.1(9)$

$\mathrm{C} 20-\mathrm{C} 15-\mathrm{C} 16-\mathrm{C} 17 \quad 1.2(10)$

$\mathrm{C} 13-\mathrm{C} 15-\mathrm{C} 16-\mathrm{C} 17 \quad 179.2(7)$

$\mathrm{C} 15-\mathrm{C} 16-\mathrm{C} 17-\mathrm{C} 18 \quad-1.6(11)$

$\mathrm{C} 16-\mathrm{C} 17-\mathrm{C} 18-\mathrm{C} 19 \quad 0.4(11)$

$\mathrm{C} 17-\mathrm{C} 18-\mathrm{C} 19-\mathrm{C} 20 \quad 1.1(9)$

$\mathrm{C} 16-\mathrm{C} 15-\mathrm{C} 20-\mathrm{C} 19 \quad 0.4(9)$

$\mathrm{C} 13-\mathrm{C} 15-\mathrm{C} 20-\mathrm{C} 19 \quad-177.7$ (5)

$\mathrm{C} 16-\mathrm{C} 15-\mathrm{C} 20-\mathrm{Te} 2 \quad-178.1(5)$

$\mathrm{C} 13-\mathrm{C} 15-\mathrm{C} 20-\mathrm{Te} 2 \quad 3.7(7)$

$\mathrm{C} 18-\mathrm{C} 19-\mathrm{C} 20-\mathrm{C} 15 \quad-1.6(8)$

$\mathrm{C} 18-\mathrm{C} 19-\mathrm{C} 20-\mathrm{Te} 2 \quad 176.8$ (4)

$\mathrm{Te} 2-\mathrm{C} 21-\mathrm{C} 22-\mathrm{C} 23 \quad 175.1(5)$

$\mathrm{C} 21-\mathrm{C} 22-\mathrm{C} 23-\mathrm{C} 24 \quad-177.3$ (7) 